Lima: Instituto de Estudios Peruano (IEP) 2008, 179 pages

\title{
Huber, Ludwig. Romper la Mano
}

\section{Carlo Mario Velarde Bazán}

\section{(2) OpenEdition}

\section{Journals}

Edición electrónica

URL: http://journals.openedition.org/bifea/3061

DOI: 10.4000/bifea.3061

ISSN: 2076-5827

\section{Editor}

Institut Français d'Études Andines

\section{Edición impresa}

Fecha de publicación: 1 diciembre 2008

Paginación: 566-568

ISSN: 0303-7495

\section{Referencia electrónica}

Carlo Mario Velarde Bazán, « Huber, Ludwig. Romper la Mano », Bulletin de l'Institut français d'études andines [En línea], 37 (3) | 2008, Publicado el 01 junio 2009, consultado el 27 noviembre 2020. URL: http://journals.openedition.org/bifea/3061 ; DOI : https://doi.org/10.4000/bifea.3061

\section{c) $(7) \ominus$}

Les contenus du Bulletin de l'Institut français d'études andines sont mis à disposition selon les termes de la licence Creative Commons Attribution - Pas d'Utilisation Commerciale - Pas de Modification 4.0 International. 


\section{Ludwig HUBER. Romper la Mano. Instituto de Estudios Peruano (IEP), Lima, 2008, 179 p.}

El libro Romper la Mano, de Ludwig Huber, representa uno de los estudios de mayor importancia en los últimos años realizado desde las Ciencias Sociales con incidencia en la Ciencia Política. El texto se encuentra dividido en cuatro capítulos, de los cuales los tres primeros representan un esfuerzo — por lo demás interesante-, para describir, presentar, hacer que se muestre el fenómeno de la corrupción, tanto como problema en general, como tema de estudio en la antropología o como legado histórico en nuestro país. Mientras que el cuarto capítulo muestra un estudio de caso de cómo opera la denominada pequeña corrupción en el departamento de Ayacucho. Este último capítulo representa uno de los pocos estudios etnográficos realizados en nuestro país sobre la práctica de la corrupción.

En el primer capítulo «El descubrimiento de la corrupción como problema», Huber nos muestra de una manera lúcida y clara los distintos enfoques teóricos y debates existentes sobre la corrupción; empezando por los revisionistas quienes «muestran» que en ciertos contextos la práctica de la corrupción puede traer efectos positivos para un país, tales como agilizar la burocracia o generar dinámicas económicas en mercados empobrecidos:

«En estas circunstancias, el soborno puede ser el lubricante para el motor de la administración pública; ayudar a ahorrar tiempo movilizando a la burocracia...» (p. 18).

Dos aspectos rescata Huber del enfoque revisionista:

«el primero es la historicidad mencionada por Leys, es decir, la observación de que lo que se considera como un acto corrupto cambia según el contexto sociohistórico. El otro aspecto, quizás más importante aún, es expresado con mayor claridad por Bayley: el referente de que la corrupción sólo es negativa en la medida que la normatividad contra la cual atenta es mejor» (p. 25).

Luego de mostrar los principales argumentos de los revisionistas, Huber presenta los principales argumentos de los moralistas quienes insisten en el carácter negativo de la práctica de la corrupción, afectando especialmente la cohesión social y provocando una situación de anomia entre los ciudadanos; y la visión economicista de la corrupción, especialmente la que el Banco Mundial propone. Finalmente Huber muestra también cómo el fenómeno de la corrupción es presentado desde la sociedad civil, especialmente por Transparencia Internacional, la cual inicialmente asume la definición propuesta por el Banco Mundial, es decir, como el uso de un cargo público para beneficio privado, sin embargo, luego amplía su definición hacia el concepto de «uso de un poder encomendado para beneficio privado» (p. 32).

Lo que hace Huber en este primer capítulo de su libro es mostrar las distintas perspectivas que tiene el fenómeno de la corrupción. Con ello, muestra algo clave de este fenómeno: que la corrupción como tal, es un fenómeno complejo, imposible de ser definido desde una sola perspectiva. Es decir, la corrupción como práctica no es unívoca. Tiene distintas formas de expresarse y por lo tanto de conceptualizarce. Asumir una sola perspectiva como completa resulta, paradójicamente, siempre un intento parcial de aproximarse a este fenómeno. En este primer capítulo, y a lo largo de todo el texto, la corrupción se presentará como un fenómeno de alta complejidad, lo cual demanda ser estudiado desde distintas disciplinas y perspectivas teóricas.

El segundo capítulo del libro aborda el fenómeno de la corrupción como práctica cultural. En ese sentido, lo que nos muestra el autor en esta sección es la complejidad del fenómeno 
enraizado en prácticas sociales y culturales que no siempre se tejen teniendo como intención llevar a cabo un acto corrupto, entendido este desde las definiciones universalistas que han ofrecido diversos teóricos e instituciones occidentales, como antes hemos visto. Por ejemplo, Huber muestra cómo los trabajos desde la antropología evidencian ciertas prácticas de construcción de redes de supervivencia o de intercambio de favores, que, leídos desde sus propios contextos socioculturales no representan necesariamente actos de corrupción. Los trabajos presentados por Huber respecto a prácticas de este tipo en Latinoamérica, África, Asia, e incluso en la propia Rusia, muestra que la definición de la corrupción ofrecida por el Banco Mundial o Transparencia Internacional resultan incompletas y por lo tanto imposible de ser tomadas en la realidad como conceptos universales.

Otro aspecto importante en este segundo capítulo es la constatación de que las ciencias sociales no han tenido como objeto de estudio sistemático la corrupción, dejando el terreno de su estudio a los enfoques jurídicos o económicos, con lo cual hace que el fenómeno de la corrupción no se permita observar de una manera comprensiva.

El tercer capítulo del libro aborda de manera muy sintética las distintas aproximaciones históricas al fenómeno de la corrupción en el Perú. Este breve recuento muestra la forma cómo el concepto de corrupción ha ido evolucionando desde la Colonia, en donde dicha práctica era relacionada al intercambio de favores de cara a obtener beneficios de la realeza, o la situación de abusos diversos de los que podían ser víctimas la población colonizada y criolla de parte de los conquistadores, hasta la situación de degeneración, en un sentido aristotélico del término, especialmente luego de la caída del régimen de Alberto Fujimori y Vladimiro Montesinos.

El último capítulo de este libro está dedicado a un estudio de caso, tal como adelantáramos al inicio de esta reseña. Ayacucho es la región elegida por nuestro autor, para mostrarnos cómo la situación de exclusión más la desconfianza en el Estado forman un caldo de cultivo importante para la aparición de la corrupción. Huber nos muestra la manera cómo la corrupción se ha constituido en un tema importante para la opinión pública general en Ayacucho, apareciendo constantemente en los medios de información regionales, especialmente la prensa, así como en el propio sentir de la población, cuando se refieren a la corrupción como el principal problema que atraviesa su sociedad.

El trabajo etnográfico realizado por nuestro autor le permite establecer cinco categorías de corrupción en la región: «Comisiones pagadas por servicios ilícitos»; «Pagos injustificados por servicios públicos regulares»; «cupos, chantaje, extorsiones»; «Nepotismo y favoritismo» y «Malversación de fondos públicos». Asimismo, le permite a Huber encontrar que las prácticas de corrupción más recurrentes se dan en el sector educación, mostrando una serie de hechos registrados como forma de escándalos en la prensa ayacuchana, o bajo la forma de denuncia realizada por la población en las instancias correspondientes respecto a ciertos hábitos de los maestros y maestras con relación a sus alumnos, las formas para lograr un cupo en una escuela pública realizando un pago indebido, o comprando puestos de docentes situados en Huamanga, cuando el puesto original ha sido asignado en una zona o comunidad lejana. Precisamente una de las formas de corrupción más frecuente que halla Huber en su trabajo de campo, tiene que ver con las horas de dictado de clases por parte de los docentes que laboran en zonas alejadas de la capital, Ilegando muchas veces a laborar solo un día a la semana, sin que por ello tengan ninguna sanción.

Otro tema importante que evidencia Huber en el desarrollo de este capítulo, es el de los discursos de los ciudadanos referentes a la corrupción. En ellos, estos se representan a sí mismos como víctimas de un flagelo que no les permite alcanzar el desarrollo moral como sociedad y el desarrollo económico como población. Sin embargo, este discurso que representa a la población como víctima entra en contradicción consigo mismo cuando la 
misma población que se percibe así acepta que la corrupción es un medio eficaz para lograr ciertos beneficios. De tal modo que la situación de víctima de la población dependerá mucho del lugar en el que se encuentre ubicado frente a la práctica de corrupción, por lo cual, la percepción de crítica es feroz cuando se hace de una manera distanciada del fenómeno, como observándolo bajo el ojo del evaluador moral de la sociedad, y será otro cuando se confronte cara a cara la posibilidad que le ofrece llevar a cabo un acto de corrupción siempre que este le depare algún beneficio personal.

El trabajo de Ludwig Huber resulta esclarecedor de muchas formas para quienes deseen aproximarse de una manera comprensiva a la práctica de la corrupción. Precisamente el aporte más interesante de este libro tal vez radique en mostrarnos que la corrupción más que ser un acto ilícito — que de hecho lo es—, representa también y sobre todo, una forma de relación entre ciudadanos y el Estado, una práctica compleja imposible de ser definida de manera absoluta. En ese sentido, cualquier intento de definición de la corrupción caerá siempre en el sesgo, en la parcialidad, cuando no en la ingenuidad teórica y práctica, si no se remite a las prácticas que le dan sentido.

Tal vez por ello las estrategias hasta ahora emprendidas por el Estado para luchar contra este vicio no ofrecen los resultados esperados. Más aún si persiste una mirada de la corrupción solo como problema jurídico o económico, y no se toma en cuenta el aspecto práctico, cultural y comprensivo de este fenómeno.

El libro Romper la Mano, representa un gran aporte para empezar una reflexión en este sentido sobre la corrupción y abre el espacio teórico y práctico para que otros profesionales de las ciencias sociales y de las humanidades empiecen a darle más atención a este complejo fenómeno. Un libro altamente recomendable. 